\title{
Chapter 10 \\ Hagedorn Model of Critical Behavior: \\ Comparison of Lattice and SBM Calculations
}

\section{Ludwik Turko}

\begin{abstract}
The Statistical Bootstrap Model and the related concept of the limiting temperature began the discussion about phase transitions in the hadronic matter. This was also the origin of the quark-gluon plasma concept. We discuss here to which extent lattice studies of QCD critical behavior at non-zero chemical potential are compatible with the statistical bootstrap model calculations.
\end{abstract}

\subsection{Rolf Hagedorn: Some Personal Impressions}

"A fireball consists of fireballs, which in turn consist of fireballs, and so on ..." that was the leading sentence from the famous CERN Yellow Report 71-12 where Rolf Hagedorn presented in detail the leading ideas and results of his Statistical Bootstrap Model (SBM) [1]. I met this report in the late 1970s having yet some scientific experience both in quantum field theory as well as in the theory of high energy multi production processes.

Starting from the beginning I realized that I was reading something unusual. I was impressed by the elegance and precision of the presentation. It was quite obvious to me that the author had spent a lot of time on discussions to clarify his arguments. Some questions were answered before I could even think about them. All was achieved without overuse of mathematical formalism, although all presentation was mathematically very rigorous. The author, however, used as simple and natural mathematical tools as possible, without going into the complex jungle of formulae and multilevel definitions. It was also clearly visible that the model, all its architecture and equipment was a one man project—Rolf Hagedorn.

And the most important point-a new idea was presented. I was not sure at that time - is this idea right or wrong - but that it was an idea not to be ignored. It was a nice answer to the long-standing question-how to effectively describe the basic structure of matter, i.e. here hadronic matter. We knew the whole hierarchy-nuclei, nucleons, elementary particles, quarks. Any of those 'levels' pretended at some time

\footnotetext{
L. Turko $(\bowtie)$
}

Institute of Theoretical Physics, University of Wrocław, Pl. Maksa Borna 9, 50-204 Wrocław, Poland 
to be the 'real' elementary one. The SBM didn't try to answer the question about basic constituents. It just pointed out that this would be a wrong question.

About 2 years later I met Rolf Hagedorn at CERN in 1979. I was quite convinced at that time about the idea of statistical bootstrap. I saw SBM as a good topic to explore - at least as a way of thinking, and, I wanted to gain a deeper knowledge about statistical physics which in the domain of strong interactions had been to me a rather obscure subject. I also convinced my Ph.D. student at that time, Krzysztof Redlich, that this mixture of statistical physics and theory of elementary particles could be a very fruitful and interesting subject.

Traveling to CERN I was quite excited to meet the physicist whose papers were giving me not only scientific but also quite aesthetic experience. In short: personal meetings with Hagedorn were even more interesting then reading his papers. He was a man of great general culture, very polite but also expecting wellprepared arguments in discussions. From the other side he was very open to share his reasoning, his calculations - even those that still were at a preliminary level of development. His handwritten notes were famous-in an almost calligraphic script, nicely written formulae, alternative arguments. He handed those notes to collaborators-it was just like received a chapter of an advanced textbook.

After 2 years our relationship rapidly changed. The martial law, introduced in Poland in December 1981, not only made impossible my stay at CERN expected in Spring 1982, but also put me first in an internee camp, then in jail. I was not the only scientist at that time who found himself in such an unexpected surrounding. And it was Rolf Hagedorn, who without any delay in the very first days of martial law, co-initiated at CERN a campaign to free interned or jailed physicists in Poland. Posters with photos and names were posted on walls of TH division, signatures of protest were collected, and letters of protest were sent to Polish officials.

When we met again in 1989 we still kept our relationship, not only on a scientific but also on a friendly level. Looking now back I must admit that Rolf Hagedorn was among those who shaped my profile - not only as a scientist, but also as a man. He was definitely worth following - in any respect. I am happy I had the possibility to be close to such an exceptional scientist and an exceptional man. A man of honor.

\subsection{Critical Behavior of Hadronic Matter}

Quantum chromodynamics (QCD) gauge theory is an excellent tool for description of single hadronic events in vacuum. However, for the dense and hot hadronic matter the most reliable theoretical results, based on first principles, can be obtained only through lattice gauge theory calculations. In particular, a phase transition or crossover phenomena are expected. This critical behavior is related to peculiarities in standard lattice QCD quantities as the Polyakov loop or susceptibilities.

From the other side, a surprisingly simple resonance gas model provides a good description of particle yields in the relativistic heavy ion collisions in the broad energy range $[2,3]$. The clue to this result is in the exponential-like behavior of the 
particle mass spectrum. This model, slightly extended [4], also reproduces results of the transition between a hadron resonance gas phase and the quark gluon plasma obtained in course of QCD lattice simulation.

The concept of the limiting temperature of the hadronic matter has appeared in the SBM [5-8]. An introduction of the baryonic chemical potential transforms this critical temperature into the critical curve, see Chap. 23. Higher internal symmetries lead to an appearance of the critical surface $[9,10]$. The hadronic matter, above the critical curve, is interpreted nowadays as a QGP phase.

I will compare here calculations of critical curves obtained in the $\mu-T$ plane from lattice Monte Carlo simulations with analogous critical curves obtained with the same input from the SBM. It was shown $[11,12]$ that a gas of non-interacting resonances provides a good description of the low temperature phase of lattice QCD. As the hadronic mass spectrum is similar to the exponential mass spectrum expected from the SBM, it is interesting to check if the critical behavior obtained from the SBM resembles Monte Carlo results of lattice QCD.

We are interested here in the region of the $\left(\mu_{B}, T\right)$ plane covered by ultrarelativistic heavy ion collisions where the phase transition is expected i.e. the high temperatures and low baryonic densities. The efficient method of lattice simulation proceeds here via a Taylor expansion with respect to the baryonic chemical potential at $\mu_{B}=0[13,14]$. This lattice technique, supplemented with other technical tools specific for QCD lattice simulations, was used to obtain the phase transition curve $T_{c}(\mu)$ for 2-flavor and 3-flavor QCD [15-19].

\section{Critical Curve from the Lattice Calculations}

In order to compare the SBM with lattice results one should take into account that the latter are not obtained from calculations performed with the physically realized quark mass spectrum. One finds [20-22] that the quark mass dependence is well parameterized through the relation

$$
\left(m_{H} a\right)^{2}=\left(m_{H} a\right)_{\text {phys }}^{2}+b\left(m_{\pi} a\right)^{2},
$$

where $\left(m_{H} a\right)_{p h y s}$ denotes the physical mass value of a hadron expressed in lattice units and $\left(m_{H} a\right)$ is the value calculated on the lattice for a certain value of the quark mass or equivalently a certain value of the pion mass.

The lattice constant $a$ can be treated as a specific ultra-violet regularization which is removed in the continuous limit $a \rightarrow 0$. The value of the critical temperature $T_{c}$ is dependent on the pion mass [17]. Pion here is understood as the lowest pseudoscalar mesonic state $q \bar{q}$ of the mass $m_{P S}$. This mass decreases to its physical pion mass $m_{\pi}=0.140 \mathrm{GeV}$ in the continuous limit along with the critical temperature.

Critical curves for 2-flavor and 3-flavor QCD were obtained at some assumed quarks masses (in lattice constant $a$ units): $m_{q}=0.1$ on the left-hand figure and $m_{q}=0.1, m_{q}=0.005$ on the right-hand plots. Corresponding $m_{P S}$ masses were $0.770 \mathrm{GeV}, 0.190 \mathrm{GeV}$ and $0.170 \mathrm{GeV}$, respectively. 


\section{Critical Curve from the Statistical Bootstrap Model}

Let us start from the bootstrap equation taken for the system with pions and nucleons taken as basic constituents. The bootstrap input function is given as, compare Eq. (27.19) on page 352

$$
\varphi_{n_{\pi}, n_{N}}(\mu, T)=2 H \pi T\left[n_{\pi} m_{\pi} K_{1}\left(\frac{m_{\pi}}{T}\right)+n_{N} m_{N} K_{1}\left(\frac{m_{N}}{T}\right) \cosh \left(\frac{\mu_{B}}{T}\right)\right],
$$

I restricted the set of input particles for a given valence quark input to the lightest mesonic and baryonic states respectively. $n_{\pi}$ and $n_{N}$ are their numbers, spin degeneration and antibaryons are taken into account here. They form an input for the SBM. So for two quark flavors there are $n_{\pi}=3$ mesonic states and $n_{N}=8$ baryonic states. For three quark flavors with the threefold quark mass degeneracy one gets $n_{\pi}=8$ and $n_{N}=32$, respectively.

The bootstrap constant $H$ is written as

$$
H=A \frac{2 m_{\pi} m_{N}}{(2 \pi)^{3}} \frac{1}{B}
$$

where $B^{1 / 4} \approx 0.190 \mathrm{MeV}$ is the bag constant to reproduce critical energy density $\varepsilon \approx 0.6 \mathrm{GeV} / \mathrm{fm}^{3}$ and the parameter $A$ is chosen so to get the critical temperature $T_{c}$ at $\mu_{B}=0$ from the corresponding QCD lattice simulation.

The Statistical Bootstrap Model used on the QCD lattice system has its basis components such as they appear in lattice QCD simulation. It means, particularly, nucleon mass expressed by pion mass (all in $\mathrm{GeV}$ ) as

$$
m_{N}\left(m_{\pi}\right)=0.94+\frac{m_{\pi}^{2}}{0.94}
$$

The critical curve for incompressible hadrons is obtained directly from the bootstrap equation

$$
2 \Phi=\varphi+e^{\Phi}-1
$$

which is meaningful only for

$$
\varphi \leq \ln 4-1
$$

So the critical curve $T_{c}(\mu)$ is given on the $\mu_{B}-T$ plane by the condition

$$
\varphi_{n_{\pi}, n_{N}}(\mu, T)=\ln 4-1 .
$$



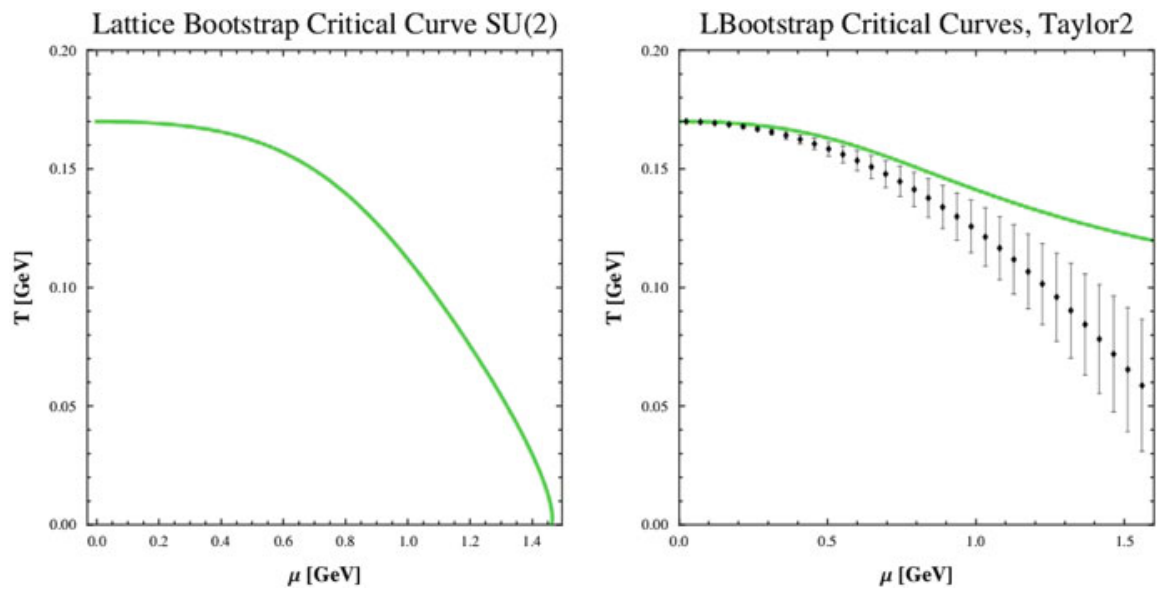

Fig. 10.1 The transition temperature $T_{c}$, as a function of baryonic chemical potential for 2-flavor lattice, bootstrap system (left-hand figure). The critical temperature 2-flavor lattice bootstrap system, compared with result of corresponding lattice simulation (right-hand figure), both considered in power law approximation

\section{Comparison of SBM and Lattice-QCD}

I consider the lattice bootstrap system as described above. The exact result is shown in left panel of Fig. 10.1, corresponding to Hagedorn's result Fig. 25.3 on page 303. This result should be compared to the 2-flavor QCD lattice simulation, indicated with vertical error line domain in the right panel in Fig. 10.1.

As the critical curves $T_{c}\left(\mu_{q}\right)$ from the QCD lattice calculations were obtained up to $\mathscr{O}\left(\left(\mu_{q} / T_{c}(0)\right)^{2}\right)$ term, so the similar approximation should be used for the critical curves obtained from Eq. (10.6). This means that the expression $\cosh \left[\left(\frac{\mu_{B}}{T}\right)\right]$ in Eq. (10.2) should be replaced by the corresponding Taylor expansion truncated to the first two terms. The result of this procedure is presented on the Fig. 10.1—right panel.

\subsection{Conclusions}

Results presented on Fig. 10.1 show that the Statistical Bootstrap Model reproduces at least qualitatively basic properties of the critical curve obtained in the course of QCD lattice simulation. We have quantitative agreement for smaller values of baryonic chemical potential, not exceeding $0.7 \mathrm{GeV}$. This is rather natural taking into account that the method used in the simulations was based on the idea of analytical continuation in the chemical potential variable, starting from the point $\mu=0$. 
The statistical bootstrap model, created by Rolf Hagedorn half a century ago, at a time when quarks were still a bold hypothesis, remains a very inspiring research tool of hadronic matter. Based on Hagedorn's deep knowledge and great intuition, the Statistical Bootstrap Model has still some unknown and unexpected properties, waiting to be discovered.

Acknowledgements I acknowledge the stimulating discussions with F. Karsch and K. Redlich. This work has been supported by the Polish National Science Center under grant no. DEC2013/10/A/ST2/00106.

Open Access This book is distributed under the terms of the Creative Commons Attribution Noncommercial License which permits any noncommercial use, distribution, and reproduction in any medium, provided the original author(s) and sources are credited.

\section{References}

1. R. Hagedorn, Thermodynamics of strong interactions. CERN Report 71-12 (1971)

2. J. Cleymans, K. Redlich, Phys. Rev. Lett. 81, 5284 (1998)

3. P. Braun-Munzinger, D. Magestro, K. Redlich, J. Stachel, Phys. Lett. B 518, 41 (2001)

4. D. Blaschke, A. Dubinin, L. Turko, Mott-hadron resonance gas and lattice QCD thermodynamics (arXiv:1501.00485 [hep-ph]) (2015)

5. R. Hagedorn, Suppl. Nuovo Cimento 3, 147 (1965); R. Hagedorn, J. Ranft, Suppl. Nuovo Cimento 6, 169 (1968)

6. R. Hagedorn, J. Rafelski, From Hadron gas to quark matter II, in Statistical Mechanics of Quarks and Hadrons, ed. by H.Satz (North Holland, Amsterdam, 1981), pp. 237-272

7. S. Frautschi, Phys. Rev. D 3, 2821 (1971)

8. R. Hagedorn, The long way to the statistical bootstrap model, Chapter 17 in this volume.

9. K. Redlich, L. Turko, Z. Phys. C 5, 201 (1980)

10. L. Turko, Phys. Lett. B 104, 153 (1981)

11. F. Karsch, K. Redlich, A. Tawfik, Eur. Phys. J. C 29, 549 (2003); Phys. Lett. B 571, 67 (2003)

12. F. Karsch, Prog. Theor. Phys. Suppl. 153, 106 (2004)

13. R.V. Gavai, S. Gupta, Phys. Rev. D 64, 074506 (2001); Phys. Rev. D 65, 094515 (2002); R.V. Gavai, S. Gupta, P. Majumdar, Phys. Rev. D 65, 054506 (2002)

14. S. Gottlieb et al., Phys. Rev. D 38, 2888 (1988)

15. C.R. Allton, S. Ejiri, S.J. Hands, O. Kaczmarek, F. Karsch, E. Laermann, C. Schmidt, L. Scorzato, Phys. Rev. D 66, 074507 (2002)

16. F. Karsch, E. Laermann, C. Schmidt, Phys. Lett. B 520, 41 (2001)

17. F. Karsch, E. Laermann, A. Peikert, Phys. Lett. B 478, 447 (2000); Nucl. Phys. B 605, 579 (2001)

18. F. Karsch, C.R. Allton, S. Ejiri, S.J. Hands, O. Kaczmarek, E. Laermann, C. Schmidt, Nucl. Phys. Proc. Suppl. 129, 614 (2004)

19. K. Redlich, F. Karsch, A. Tawfik, J. Phys. G 30, S1271 (2004)

20. D.G. Richards et al. [LHPC Collaboration], Nucl. Phys. Proc. Suppl. 109A, 89 (2002)

21. M. Göckeler et al., Phys. Lett. B 532, 63 (2002)

22. J.M. Zanotti et al., [CSSM lattice collaboration], Phys. Rev. D 68, 054506 (2003) 